\title{
ON THE HEDGING PORTFOLIO OF ASIAN OPTIONS
}

\author{
MiCHEL JACQUES \\ École d'actuariat, Université Laval
}

\begin{abstract}
We give 2 explicit formulae for the hedging portfolio of Asian options. One is based on the usual Lognormal approximation, and the other on an Inverse Gaussian approximation. Both give excellent results as replicating strategies when the parameters of the model are in a reasonable range.
\end{abstract}

\section{KEYWORDS}

Option pricing theory; Asian option; replicating strategy; hedging portfolio.

\section{INTRODUCTION}

Asian options are path-dependent contingent claims since they settle against some kind of average of the quoted stock prices over a prescribed period. In the case where the average is the continuous (integral) average, Geman and Yor (1993) have obtained an expression for the Laplace transform of the price. The inverse Laplace transform however has to be taken numerically. In the case where the average is the discrete geometric average, exact expressions of the Black-Scholes type can be obtained (Kemna and Vorst (1990)).

In this paper we consider the case where the average is the (discrete) arithmetic average of the last $n$ prices. The challenge with these options is that the distribution of the average is not known when we make the usual assumption that the stock price follows a geometric Brownian motion. Up to now there is no way of computing exactly the price of these Asian options. Also, since the payoff considered here is different from the payoff of continuous average options, we should be careful if we were to approximate the price of the arithmetic average option by the price of the continuous average option. This is not what we are going to do here, since we prefer a more direct approach (of the Black-Scholes type) to a numerical inversion of a Laplace transform.

Different approaches for the evaluation of the price of (arithmetic average) Asian options have been taken. First Kemna and Vorst (1990) used Monte-Carlo simulation. This is however time-consuming and does not lead to the hedging portfolio. A faster approach based on an Edgeworth expansion around the Lognormal distribution has 
been given by Turnbull and Wakeman (1991). Levy (1992) remarked that the higher terms in the Edgeworth expansion have a negligible numerical value for reasonable values of the parameters of the model and proposed to simply use a Lognormal approximation for pricing, giving closed-form formulae for the approximate price.

The aim of the present paper is to extend this approximate analysis to the construction of the hedging portfolio. If we had an exact formula for the price, standard delta hedging would provide a dynamical replicating strategy (Bergman (1985)). This is a rule indicating how to invest the price of the option so that the investment reproduces the value of the option at maturity. Since we have here an approximate formula for the price, we propose to study the accuracy of the approximation by computing the replicating strategy and by comparing its maturity value with the maturity value of the option. By the same procedure, we also remark that an approximation of the distribution of the average by an Inverse Gaussian distribution gives results comparable to those of the Lognormal approximation.

The paper is organized as follows. We first recall the recursive computation of the first two moments of the arithmetic average and then give evidence that an Inverse Gaussian approximation is just as good as a Lognormal approximation. Using these two approximations we derive explicit formulae for the hedging portfolio and show through numerical examples that these formulae are efficient in the sense that the replicating strategy is close to the intrinsic value of the option at maturity time.

\section{COMPUTATION OF THE MOMENTS}

We assume that there is on the market a stock with price described by a stochastic process $\{S(t), t \geq 0\}$ following a geometric Brownian motion:

$$
d S(t)=a S(t) d t+b S(t) d B(t),
$$

where $\{B(t), t \geq 0\}$ is a standard Brownian motion. The parameter $a$ is the mean rate of return of the stock and the parameter $b$ is called its volatility. There is also on the market a risk-free instrument (the bond) with a constant rate of return $r$. We also assume that the bond and stock may be purchased in any (fractional) amount and that there is no restriction on short sales. By arbitrage arguments (Harrison and Pliska (1981), Harrison and Kreps (1979)), we have to change the probability measure so that $a=r$ in all pricing computations.

The Asian options considered in this paper are derivatives which settle at a future date $T$ against the arithmetic average of the last $n$ quoted prices of the stock. The payoff of the option is thus

$$
\max \left\{0, \frac{S(T-n+1)+\ldots+S(T)}{n}-K\right\},
$$

where $K$ is the option strike price. In the sequel, we assume that time is measured in units so that $T$ is integral, hence the quoted prices are taken at integral times.

Because of (1), the random variable $S(t)$ has a Lognormal distribution. The distribution of the sum of (dependent) Lognormal distributions is at present not known. 
However, the moments of such a sum can be computed in a recursive way. Computing the moments directly by summing up the terms becomes complicated due to the dependence of the terms. An easy way of avoiding this is to work backwards, starting with the last summand and to isolate the (independent) increments of Brownian motion. This approach has been taken in Turnbull and Wakeman (1991) and is given here in order to fix notations.

Let

$$
Y(r, b, n, T)=\frac{S(T-n+1)+\ldots+S(T)}{n}
$$

be the arithmetic average of the last $n$ stock prices. In the present section, we compute the moments of $Y$, given that $S(t)=x$ with $t<T-n+1$. (The case $t \geq T-n+1$ can be treated along the same lines, see section 4.) We have

$$
\begin{aligned}
Y(r, b, n, T) \\
=\frac{1}{n} S(T-n+1)\left[1+\frac{S(T-n+2)}{S(T-n+1)}+\ldots+\frac{S(T)}{S(T-n+1)}\right] \\
=\frac{1}{n} S(T-n+1)\left[1+\exp \left(\left(r-\frac{b^{2}}{2}\right)+b N_{T-n+2}\right)+\ldots\right. \\
\left.+\exp \left(\left(r-\frac{b^{2}}{2}\right)+b N_{T-n+2}+\ldots+\left(r-\frac{b^{2}}{2}\right)+b N_{T}\right)\right] \\
=\frac{1}{n} S(T-n+1) \\
{\left[1+\exp \left(\left(r-\frac{b^{2}}{2}\right)+b N_{T-n+2}\right)\left[1+\exp \left(\left(r-\frac{b^{2}}{2}\right)+b N_{T-n+3}\right)\right.\right.} \\
\\
{\left.\left[\ldots 1+\exp \left(\left(r-\frac{b^{2}}{2}\right)+b N_{T-1}\right)\left[1+\exp \left(\left(r-\frac{b^{2}}{2}\right)+b N_{T}\right)\right] \ldots\right]\right] }
\end{aligned}
$$

where $\left\{N_{\mathrm{t}}, t=T-n+2, \ldots, T\right\}$ are standardized Normal variables which are independent due to the independence of the increments of Brownian motion. Let then

$$
\begin{aligned}
& Y_{0} \equiv 1+\exp \left(\left(r-\frac{b^{2}}{2}\right)+b N_{T}\right) \\
& Y_{k} \equiv 1+\exp \left(\left(r-\frac{b^{2}}{2}\right)+b N_{T-k}\right) Y_{k-1},
\end{aligned}
$$

for $k=1 \ldots n-2$. Note that $N_{T-k}$ is independent of $Y_{k-1}$. We are interested in

$$
Y(r, b, n, T)=\frac{1}{n} S(T-n+1) Y_{n-2} .
$$


Note again that $S(T-n+1)$ is independent of $Y_{n-2}$. Taking the first two moments of $(2-4)$, we get

$$
\begin{aligned}
\mathrm{E}\left[Y_{0}\right] & =1+e^{r}, \\
\mathrm{E}\left[Y_{0}^{2}\right] & =1+2 e^{r}+e^{2 r+b^{2}}, \\
\mathrm{E}\left[Y_{k}\right] & =1+e^{r} \mathrm{E}\left[Y_{k-1}\right], \\
\mathrm{E}\left[Y_{k}^{2}\right] & =1+2 e^{r} \mathrm{E}\left[Y_{k-1}\right]+e^{2 r+b^{2}} \mathrm{E}\left[Y_{k-1}^{2}\right], \\
\mathrm{E}[Y(r, b, n, T)] & =\frac{1}{n} \mathrm{E}[S(T-n+1)] \mathrm{E}\left[Y_{n-2}\right], \\
\mathrm{E}\left[Y(r, b, n, T)^{2}\right] & =\frac{1}{n^{2}} \mathrm{E}\left[S(T-n+1)^{2}\right] \mathrm{E}\left[Y_{n-2}^{2}\right] .
\end{aligned}
$$

Proceeding recursively, or using symbolic software to solve for the recursive equations (5-8), we get

$$
\begin{aligned}
\mathrm{E}\left[Y_{n-2}\right] & =\frac{e^{r n}-1}{e^{r}-1}, \\
\mathrm{E}\left[Y_{n-2}^{2}\right] & =\frac{A(r, b, n)}{\left(e^{r}-1\right)\left(e^{2 r+b^{2}}-1\right)\left(e^{r}-e^{2 r+b^{2}}\right)},
\end{aligned}
$$

where the numerator $A(r, b, n)$ is given by the expression:

$$
\begin{aligned}
A(r, b, n)= & -2 e^{r(n+1)}+2 e^{r(n+3)+b^{2}}+e^{2 r} \\
& -e^{2 r(n+1)+b^{2} n}+e^{r(2 n+1)+b^{2} n}-e^{r(2 n+3)+b^{2}(n+1)} \\
& -e^{3 r+b^{2}}+e^{r}+e^{\left(2 r+b^{2}\right)(n+1)}-e^{2 r+b^{2}} .
\end{aligned}
$$

Let now $m_{I}(x, t, r, b, n)$ and $m_{2}(x, t, r, b, n)$ be the first and second moment of the arithmetic average of $n$ quoted prices of the stock, computed $t$ periods before the first price to enter in the arithmetic mean, given that the current stock price is $x$. Using (912), we immediately get

$$
\begin{aligned}
& m_{1}(x, t ; r, b, n)=\frac{x e^{r t}\left(e^{r n}-1\right)}{n\left(e^{r}-1\right)} \\
& m_{2}(x, t ; r, b, n)=\left(\frac{x}{n}\right)^{2} \frac{e^{\left(2 r+b^{2}\right) t} A(r, b, n)}{\left(e^{r}-1\right)\left(e^{2 r+b^{2}}-1\right)\left(e^{r}-e^{2 r+b^{2}}\right)}
\end{aligned}
$$

We will use the two explicit formulae (13) and (14) in order to obtain theoretical results in the sequel, but we will not use them for numerical evaluation, since (14) gives a ratio of extremely small numbers for reasonable values of the parameters $r$ and $b$ and is thus numerically unusable. The expression (14) could be rewritten in an algebraically equivalent form which is numerically usable. This form is given in the appendix of Levy (1992). However, we found it as easy to use the recursive formulae (5-10) to numerically evaluate the moments. 


\section{TWO APPROXIMATIONS TO THE DISTRIBUTION OF THE ARITHMETIC MEAN}

Since the distribution of $Y(r, b, n, T)$ is not known, we have to use some kind of approximation based on our knowledge of the moments. Levy (1992) gave statistical tests on the third and fourth moments in order to substanciate the Lognormal approximation. One interesting point is that the recursive equation (3) is similar to the recursive equation used in Risk Theory to compute the distribution of the total portfolio claim in the individual model (Bowers et al. (1986)). The difference is that equation (3) is a relation between the independent variables $\mathrm{N}_{T-k}$ and $\mathrm{Y}_{k-1}$ which is more complicated than the usual sum of independent variables of Risk Theory. However it is natural to consider other approximations as is usually done in Risk Theory.

Different choices of distributions have been tried, most of them giving obviously bad results for the option price. However the approximation of the distribution of the arithmetic average by an Inverse Gaussian distribution (IG) gives prices comparable to those given by the Lognormal approximation ( $\mathrm{LN})$ when the parameters are chosen in the same range as in Levy (1992), Kemna and Vorst (1990) or Turnbull and Wakeman (1991).

Table 1 gives the price of an option computed with the formulae given later in the paper (see (29) and (46)), based on the two approximations. The parameters in Table 1 are chosen as an initial stock price $S(0)=100$, an annual (nominal, daily compounded) interest rate of $9 \%$ (i.e. $r=\ln \left(1+\frac{0.09}{365}\right)$ daily), a maturity $T$ of 120 days and an averaging period $n$ of 30 days. The values of the volatility $b$ are also on an annual basis. Some of these numerical values may be considered as being high nowadays, but they are chosen to be in the same range as in the papers cited above.

TABLE 1

LN AND IG APPROXIMATE OPTION PRICE

\begin{tabular}{crrr}
\hline $\boldsymbol{b}$ & $\boldsymbol{K}$ & LN & \multicolumn{1}{c}{ IG } \\
\hline 0.2 & 90 & 12.68 & 12.68 \\
& 100 & 5.46 & 5.46 \\
0.3 & 110 & 1.63 & 1.63 \\
& 90 & 13.85 & 13.85 \\
& 100 & 7.48 & 7.49 \\
0.4 & 110 & 3.48 & 3.49 \\
& 90 & 15.36 & 15.37 \\
& 100 & 9.51 & 9.53 \\
& 110 & 5.48 & 5.49 \\
\hline
\end{tabular}

As we see from Table 1, the agreement between the prices computed according to the two approximations is excellent. We want to give further evidence for the use of these approximations by testing directly the distribution and not only its moments. This can be done by measuring the distance between a simulated sample of the true (unknown) distribution of the arithmetic mean and the distribution of the approximating variable. 
First, let us recall that $X_{1}$ has a Lognormal distribution with parameters $\mu$ and $\sigma$ $(\mathrm{LN}(\mu, \sigma)$ in short) if its density is

$$
f_{X_{1}}(x)=\frac{\exp \left(-\frac{1}{2}\left(\frac{\ln x-\mu}{\sigma}\right)^{2}\right)}{x \sigma \sqrt{2 \pi}},
$$

for $x>0$. A straightforward calculation gives

$$
\begin{aligned}
& \mathrm{E}\left[X_{1}\right]=e^{\mu+\sigma^{2} / 2}, \\
& \mathrm{E}\left[X_{1}^{2}\right]=e^{2 \mu+2 \sigma^{2}} .
\end{aligned}
$$

On the other hand, $X_{2}$ has an Inverse Gaussian distribution with parameters $\rho$ and $\beta$ $(\operatorname{IG}(\rho, \beta)$ in short) if its density is

$$
f_{X_{2}}(x)=\frac{\rho \exp \left(-\frac{(x-\rho)^{2}}{2 \beta x}\right)}{\sqrt{2 \pi x^{3} \beta}},
$$

for $x>0$. This distribution (with an appropriate choice of parameters) appears as the distribution of the first time when Brownian motion hits a barrier. The reader may consult Johnson and Kotz (1970), chapter 15 for more information. Up to a transformation of parameters, the above density is equation (4.1), page 138 of that reference. A straightforward calculation gives

$$
\begin{aligned}
& \mathrm{E}\left[X_{2}\right]=\rho, \\
& \mathrm{E}\left[X_{2}^{2}\right]=\beta \rho+\rho^{2} .
\end{aligned}
$$

The approximations are obtained by identifying the first two moments of both distributions with the first two moments of $Y(r, b, n, T)$. We thus choose the parameters as

$$
\begin{aligned}
\mu & =2 \ln m_{1}-\frac{1}{2} \ln m_{2}, \\
\sigma^{2} & =\ln m_{2}-2 \ln m_{1},
\end{aligned}
$$

for the Lognormal approximation and

$$
\begin{aligned}
& \rho=m_{1}, \\
& \beta=\frac{m_{2}-m_{1}^{2}}{m_{1}},
\end{aligned}
$$

for the Inverse Gaussian approximation. Notice that $m_{1}$ and $m_{2}$ are shorthand for $m_{l}(S(0), T-n+1 ; r, b, n)$ and $m_{2}(S(0), T-n+1 ; r, b, n)$ and, similarly, the 4 parameters $\mu, \sigma, \rho$ and $\beta$ are functions of $(S(0), T-n+1 ; r, b, n)$.

We then proceed as follows. We generate $N$ trajectories of the geometric Brownian motion (stock price) and record for each trajectory the value of the arithmetic average of the last $n$ prices. This gives a sample of size $N$ of $Y(r, b, n, T)$, say $\left\{y_{l}, \ldots, y_{N}\right\}$. We can measure the distance between the cumulative distribution $F_{N}$ of this sample of the 
exact distribution and the cumulative distribution $F$ of either a $\mathrm{LN}$ or IG variable by a kind of Cramér-von Mises distance:

$$
d_{1}=\sum_{i=1}^{N}\left(F_{N}\left(y_{i}\right)-F\left(y_{i}\right)\right)^{2} .
$$

(See Hogg and Klugman (1984), page 83). In the computation of the option price, the small values of $Y$ (i.e. values less than $K$ ) do not appear. We might thus want to give more weight to the right tail of the distribution and use a weighted Cramér-von Mises distance:

$$
d_{2}=\sum_{i=1}^{N} \frac{\left(F_{N}\left(y_{i}\right)-F\left(y_{i}\right)\right)^{2}}{1-F\left(y_{i}\right)}
$$

For $N=10,000$ and the same numerical values as in Table 1 , the values of the two distances for the two approximations are given in Table 2.

TABLE 2

DISTANCE BETWEEN APPROXIMATE AND EXACT DISTRIBUTIONS

\begin{tabular}{cccccc}
\hline & & \multicolumn{2}{c}{$\boldsymbol{d}_{\mathbf{1}}$} & & \multicolumn{2}{c}{$\boldsymbol{d}_{\mathbf{2}}$} \\
\hline $\boldsymbol{b}$ & LN & IG & LN & IG \\
\hline 0.2 & 0.42 & 0.43 & 0.89 & 0.89 \\
0.3 & 0.43 & 0.44 & 0.92 & 0.92 \\
0.4 & 0.28 & 0.27 & 0.78 & 0.85 \\
\hline
\end{tabular}

It is clear from Table 2 that the two approximating distributions are at the same distance from the distribution of the sample and this partially explains that the prices computed according to the two approximations are close to each other. Other candidate distributions have been tried and the corresponding distances are substantially larger. As a point of reference, the distances computed with a Normal distribution are 10 to 30 times larger than the distances reported in Table 2. The conclusion is that the IG approximation should thus be considered on the same footing as the usual LN approximation.

The above values of the distances have no absolute meaning and thus do not provide a measure of the quality of the approximations. However, if we make the assumption that $Y$ has one of the two approximate distributions, we can compute the hedging portfolio explicitly. Then we can check how well this portfolio replicates the value of the option along a random trajectory of the stock price. Pricing of options is performed in an arbitrage-free setting and arbitrage-free evaluation is tantamount to the existence of a replicating strategy. We thus propose to assess the quality of the approximations by using the very key ingredient of option pricing theory. 


\section{THE REPLICATING STRATEGY IN THE LOGNORMAL APPROXIMATION}

Let $u(x, t ; r, b, n, T, K)$ be the price of the option sold at time $t$, given that the price of the stock is $S(t)=x$. If we build the replicating strategy determined by delta hedging, we need

$$
\xi(x, t ; r, b, n, T, K) \equiv \frac{\partial u}{\partial x}(x, t ; r, b, n, T, K)
$$

Defining now $\eta$ by

$$
\eta \equiv \frac{u(x, t ; r, b, n, T, K)-\xi x}{S(0) e^{r t}}
$$

we can interpret $\xi$ as the number of shares of stock and $\eta$ as the number of shares of the bond (measured in units of $S(0)$ ), since we have:

$$
u(x, t ; r, b, n, T, K)=\xi x+\eta S(0) e^{r t} .
$$

By arbitrage arguments (Harrison and Pliska (1981), Harrison and Kreps (1979)), it is well-known that the price $u(x, t, r, b, n, T, K)$ is computed as

$$
\begin{aligned}
u(x, t ; r, b, n, T, K) & =e^{-r(T-t)} \mathrm{E}[\max \{0, Y(r, b, n, T)-K\} \mid S(t)=x] \\
& =e^{-r(T-t)} \int_{K}^{\infty}(y-K) f_{Y}(y) d y,
\end{aligned}
$$

where $f_{Y}$ is the probability density of $Y$, approximated here by the density of a variable of type $\mathrm{LN}(\mu, \sigma)$. In order to compute this integral, we need to relate the parameters $\mu$ and $\sigma$ to the option parameters. For this, we distinguish 3 cases.

\subsection{The case $t<T-n+1$}

If we are before the averaging period, $\mu=\mu(x, T-t-n+1 ; r, b, n)$ and $\sigma=\sigma(x, T-$ $t-n+1 ; r, b, n)$ (see (21), (22)). A straightforward integration gives an analogue of the Black-Scholes formula:

$$
u(x, t ; r, b, n, T, K)=e^{-r(T-t)}\left[e^{\mu+\sigma^{2} / 2} \Phi\left(\frac{\sigma^{2}+\mu-\ln K}{\sigma}\right)-K \Phi\left(\frac{\mu-\ln K}{\sigma}\right)\right]
$$

where $\Phi$ is the cumulative probability function of a standardized Normal variable and the parameters $\mu$ and $\sigma$ are evaluated at $(x, T-t-n+1 ; r, b, n)$. Notice that the integration is well-known from the computation of Stop-Loss premiums. In order to get the replicating strategy, we need to differentiate $u(x, t ; r, b, n, T, K)$ with respect to $x$. The only $x$ dependence in $u$ is through the parameters $\mu$ and $\sigma$. By (13) and (14), we have

$$
\begin{aligned}
& \frac{\partial m_{1}(x, t ; r, b, n)}{\partial x}=\frac{m_{1}(x, t ; r, b, n)}{x}, \\
& \frac{\partial m_{2}(x, t ; r, b, n)}{\partial x}=\frac{2 m_{2}(x, t ; r, b, n)}{x},
\end{aligned}
$$


and we easily get, using (21) and (22), that

$$
\begin{gathered}
\frac{\partial \mu(x, t ; r, b, n)}{\partial x}=\frac{1}{x}, \\
\frac{\partial \sigma(x, t ; r, b, n)^{2}}{\partial x}=0 .
\end{gathered}
$$

Differentiating (29) with (32) and (33) and simplifying, we have

$$
\xi(x, t ; r, b, n, T, K)=e^{-r(T-t)} \frac{1}{x} e^{\mu+\sigma^{2} / 2} \Phi\left(\frac{\sigma^{2}+\mu-\ln K}{\sigma}\right),
$$

where the parameters $\mu$ and $\sigma$ are evaluated at $(x, T-t-n+1 ; r, b, n)$.

\subsection{The case $t \geq T-n+1, t$ non-integral}

Now some values of the stock price entering into the arithmetic mean are known. Let $\bar{t}$ be the integral part of $t$. Then

$$
\begin{aligned}
u(x, t ; r, b, n, T, K)= & e^{-r(T-t)} \mathrm{E}\left[\operatorname { m a x } \left\{0, \frac{1}{n}(S(T)+S(T-1)+\ldots\right.\right. \\
& +S(\bar{t}+1)+S(\bar{t})+\ldots+S(T-n+1))-K\} \mid S(t)=x] \\
= & e^{-r(T-t)} \frac{T-\bar{t}}{n} \mathrm{E}\left[\max \left\{0, Y^{\prime}-K^{\prime}\right\} \mid S(t)=x\right],
\end{aligned}
$$

where

$$
\begin{aligned}
Y^{\prime} & =\frac{S(T)+S(T-1)+\ldots+S(\bar{t}+1)}{T-\bar{t}}, \\
K^{\prime} & =\frac{n K-(S(T-n+1)+S(T-n+2)+\ldots+S(\bar{t}))}{T-\bar{t}} .
\end{aligned}
$$

The price is similar to the price in (28), except that the arithmetic average is now taken over $T-\bar{t}$ future prices instead of $n$ prices, the strike price is rescaled to $K$ ' and we compute the price $1-t+\bar{t}$ periods before the first price to enter into the mean. If $K^{\prime}>$ 0 , the situation is similar to that in the previous subsection and, up to a global factor, $u(x, t ; r, b, n, T, K)$ takes the same form as in (29) with $K$ replaced by $K^{\prime}$ and the parameters evaluated at $(x, 1-t+\bar{t} ; r, b, T-\bar{t})$. If $K^{\prime} \leq 0, \max \left\{0, Y^{\prime}-K^{\prime}\right\}$ is $Y^{\prime}-K^{\prime}$ and the expectation gives $\mathrm{E}\left[Y^{\prime}\right]-K^{\prime}$. Hence, if $K^{\prime} \leq 0$,

$$
u(x, t ; r, b, n, T, K)=e^{-r(T-t)} \frac{T-\bar{t}}{n}\left(e^{\mu+\sigma^{2} / 2}-K^{\prime}\right),
$$

with the parameters evaluated at $(x, 1-t+\bar{t} ; r, b, T-\bar{t})$. Differentiating with respect to $x$ thus gives

$$
\xi(x, t ; r, b, n, T, K)=e^{-r(T-t)} \frac{T-\bar{t}}{n} \frac{1}{x} e^{\mu+\sigma^{2} / 2} \Phi\left(\frac{\sigma^{2}+\mu-\ln K^{\prime}}{\sigma}\right),
$$


if $K^{\prime}>0$, and

$$
\xi(x, t ; r, b, n, T, K)=e^{-r(T-t)} \frac{T-\bar{t}}{n} \frac{1}{x} e^{\mu+\sigma^{2} / 2},
$$

if $K^{\prime} \leq 0$. In both equations the parameters $\mu$ and $\sigma$ are evaluated at $(x, 1-t+\bar{t} ; r, b, T-\bar{t})$.

\subsection{The case $t \geq T-n+1, t$ integral}

The situation is essentially the same as in the previous subsection with $\bar{t}=t$. The price $u(x, t, r, b, n, T, K)$ is as before with the parameters evaluated at $(x, 1 ; r, b, T-t)$. But now, by (37), $K$ ' contains $S(t)=x$ and is thus a function of $x$. We have immediately that

$$
\frac{\partial K}{\partial x}=-\frac{1}{T-t}
$$

We thus pick up an extra term when differentiating with respect to $x$ :

$$
\begin{aligned}
\xi(x, t ; r, b, n, T, K)= & e^{-r(T-t)} \frac{T-t}{n}\left[\frac{1}{x} e^{\mu+\sigma^{2} / 2} \Phi\left(\frac{\sigma^{2}+\mu-\ln K^{\prime}}{\sigma}\right)\right. \\
& \left.+\frac{1}{T-t} \Phi\left(\frac{\mu-\ln K^{\prime}}{\sigma}\right)\right],
\end{aligned}
$$

if $K^{\prime}>0$, and

$$
\xi(x, t ; r, b, n, T, K)=e^{-r(T-t)} \frac{T-t}{n}\left(\frac{1}{x} e^{\mu+\sigma^{2} / 2}+\frac{1}{T-t}\right),
$$

if $K^{\prime} \leq 0$. In both equations the parameters $\mu$ and $\sigma$ are evaluated at $(x, 1 ; r, b, T-t)$.

\subsection{Examples}

We give in the present subsection examples of the hedging portfolio along randomly generated trajectories of the stock price process. The portfolio described in the preceding subsections is assumed to be updated continuously. If we update it only at discrete times, we expect it to only provide an approximation to the replicating (autofinancing) strategy for the intrinsic value of the option. We present here numerical examples when the portfolio is updated 100 times between two successive quotations entering into the average. For comparison sake, we also plot on the figure the intrinsic value of the option, if it were to attain maturity at the current time. This intrinsic value is $\max \{0$, (n-periods running average) $-K\}$. For $t<n$, we need values of the stock price at negative times to compute the arithmetic average and we assume that $S(t)=S(0), t<0$. In the illustrations, we take the interest rate to be $9 \%$ per annum, the volatility to be 0.2 per annum, the maturity date to be 120 days and the averaging period to be 30 days. The initial price of the stock is 100 and the strike price of the option is 90 . 
For pricing purposes, we need to assume $a=r$, but here we consider true "physical" trajectories. We have thus to generate the trajectories taking into account the real rate of return $a$ of the stock. In the illustrations, $a$ is taken as $15 \%$, significantly higher than the risk-free rate.

We assume that the price of the stock is monitored at discrete times $\left\{t_{1}, \ldots, t_{N}\right\}$ with $N=k T$. Thus the portfolio will be updated $k$ times between two successive quotations entering into the average. The hedging portfolio at time $t_{k}$ is denoted $\pi\left(t_{k}\right)$ and is initialized at the selling price of the option:

- $\pi(0)=u(S(0), 0 ; r, b, n, T, K)$

- compute $\xi(0)$ according to (34) and buy $\xi(0)$ shares of stock

- invest (or borrow) the rest in the bond: $\eta(0)=\frac{\pi(0)-\xi(0) S(0)}{S(0)}$

Next, at each monitoring time $t_{k}$, just after the stock price is revealed,

- compute the value of the portfolio: $\pi\left(t_{k}\right)=\xi\left(t_{k-1}\right) S\left(t_{k}\right)+\eta\left(t_{k-1}\right) S(0) e^{r t_{k}}$

- compute $\xi\left(t_{k}\right)$ according to (34), (38), (39), (41) or (42) and buy $\xi\left(t_{k}\right)$ shares of stock

- invest (or borrow) the rest in the bond: $\eta\left(t_{k}\right)=\frac{\pi\left(t_{k}\right)-\xi\left(t_{k}\right) S\left(t_{k}\right)}{S(0) e^{r t_{k}}}$

Figure 1 presents a trajectory where the option ends up in the money (i.e. is exercised) while in Figure 3 the option ends up out of the money (i.e. is not exercised). The smooth curve is the current intrinsic value of the option (smoothness being caused by averaging) and the peaky curve is the value of the hedging portfolio $\pi$. The value of the option $u(x, t)$ at any time is also plotted on the figures, but it is undistinguishable from the hedging portfolio $\pi$. This means that the hedging portfolio reproduces the price of the option along the path and the intrinsic value of the option at maturity with a very good precision for those particular trajectories. It is not easy to see the improvement gained from increasing the updating frequency $k$ on a figure, so we leave this for the numerical analysis at the end of this subsection.

Figures 2 and 4 give the components of the hedging portfolio for the trajectories of Figures 1 and 3, respectively. The positive part is the stock part $\xi$, whereas the negative part is the bond part $\eta$. A negative bond means it is short-sold or borrowed. The behaviour of the two components in Figure 2 after the averaging has begun is to be noted. It is more or less linear; this seems to be characteristic of Asian options.

Hedge ratios for Asian options have also been considered by Vorst (1992), but his approximation is based on the geometric average option and is thus different from (and more complicated than) the approximation given here. Moreover he only gave the hedge ratio for the case $t<T-n+1$ and then compared the result with Monte-Carlo simulation at a particular time. This information is clearly not sufficient to construct 
Figure 1. HEDGiNG PORTFOLIO: OPTION EXERCISED

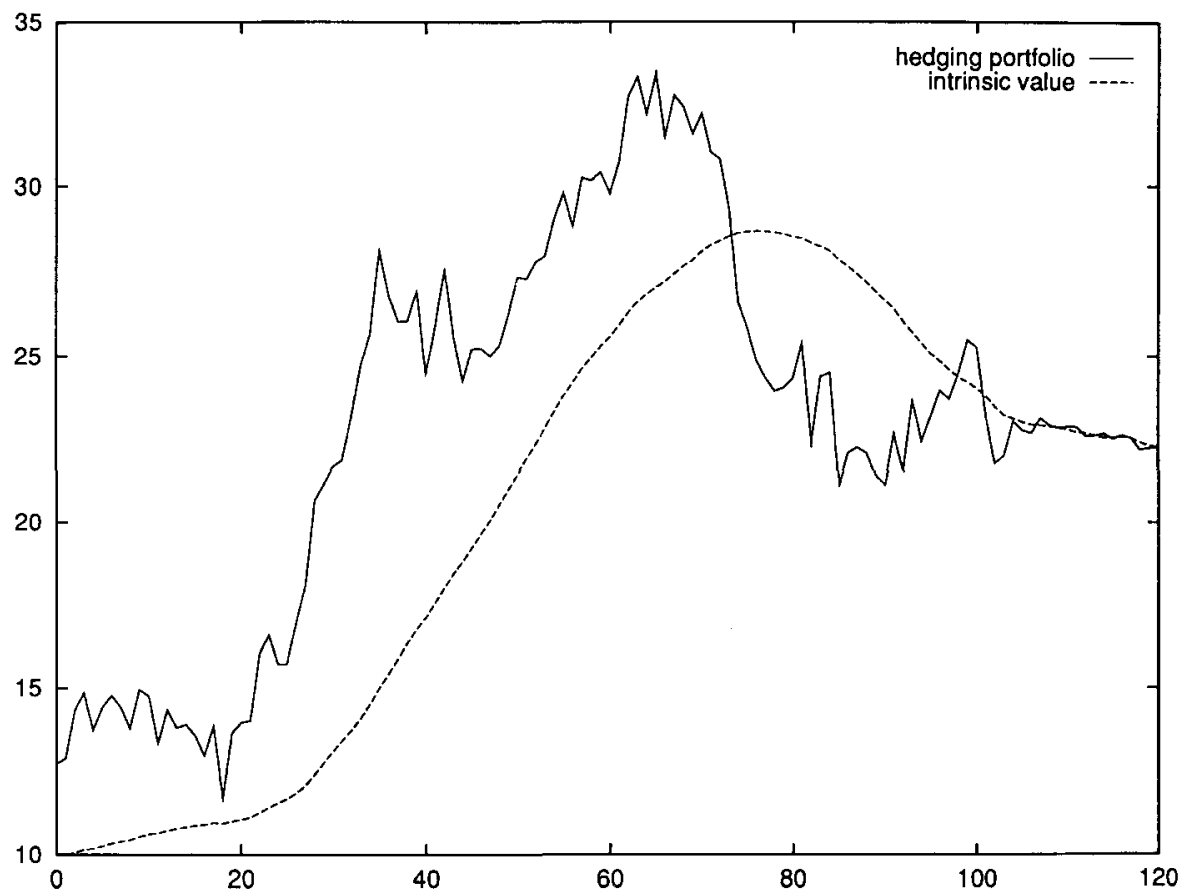

the portfolio along the whole trajectory. Since we have only approximate formulae for the hedge ratio, it is of crucial importance to check that the approximate hedging portfolio is close to the intrinsic value of the option at maturity date. Indeed the error contained in the hedge ratio at a particular time could propagate in time and the dynamically constructed portfolio could substantially deviate from the intrinsic value of the option at maturity time. It is apparent from the trajectories in Figures 1 and 3 that it is not the case for those particular trajectories.

A more detailed analysis of the accuracy of the hedging portfolio can be made. Since the intrinsic value of the option at maturity can take arbitrarily small or arbitrarily large values, a natural candidate would be the relative error of the ultimate value of the hedging portfolio with respect to the intrinsic value of the option. However this excludes the cases where the intrinsic value vanishes. We are thus going to study the absolute error of the ultimate value of the hedging portfolio with respect to the intrinsic value of the option at maturity:

$$
\epsilon=\text { hedging portfolio }- \text { intrinsic value. }
$$

We randomly generate 1,000 trajectories of the stock price process with the same numerical values of the parameters as for the figures above. We record the corresponding errors, thus providing a sample of size 1,000 for $\in$. The sample is generated with the hedging portfolio updated $k$ times between two successive quotations entering into the average, with $k=1,10,100,1000$ and 10000 . The large values of $k$ may be consi- 
FIGURE 2. COMPONENTS OF PORTFOLIO: OPTION EXERCISED

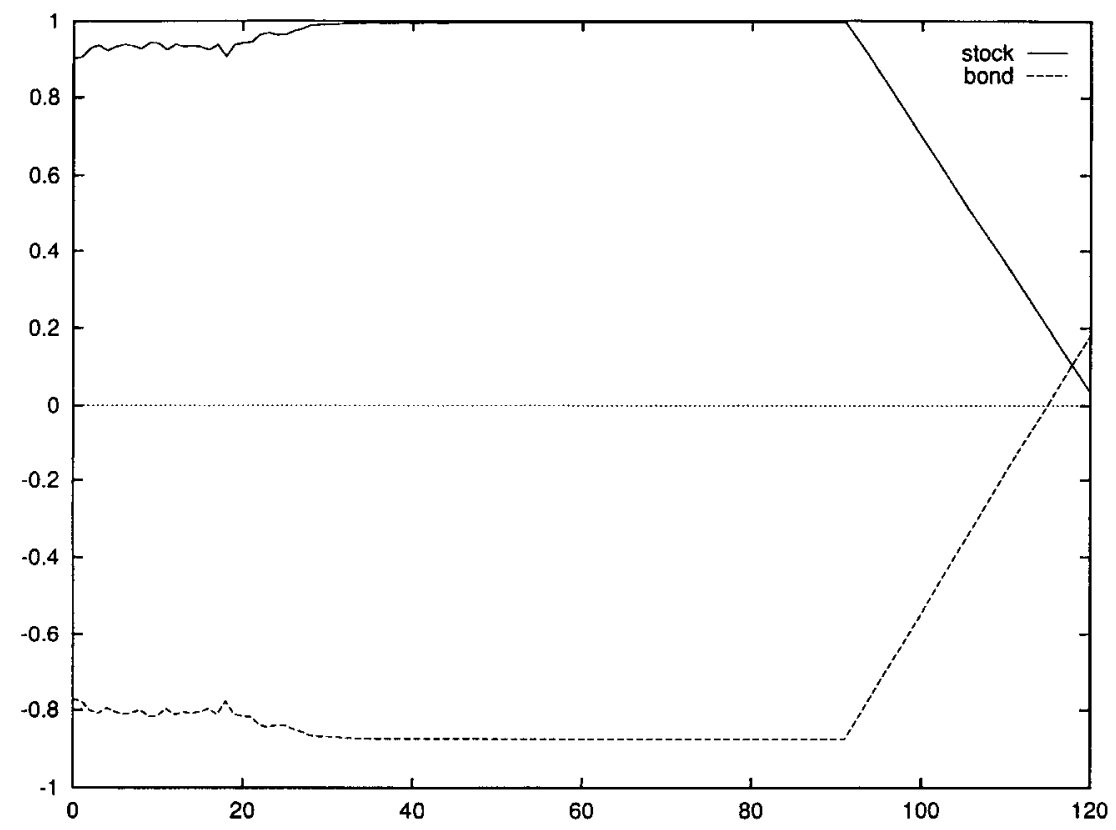

dered as excessive from a practical point of view, but the idea here is to study the quality of the approximation. In order to use the hedging portfolio for that purpose, we need in principle to update the portfolio continuously. The large values of $k$ should be seen as this continuous limit and not as a practical benchmark.

The proportion of trajectories in the sample with $|\epsilon|<0.1$ is given in the LN column of Table 3. Recall from Table 1 that the price of the option for the parameter values considered is 12.68 , meaning that the average payoff is about 10 . An error of 0.1 may then be considered as small. Smaller values of $\in$ would require larger values of the updating frequency $k$ in order to achieve good behaviour. This is also shown in Table 3 (LN column) for the proportion of trajectories with $|\in|<0.01$.

TABLE 3

ERROR ON THE HEDGING PORTFOLIO

\begin{tabular}{rrrrr}
\hline & \multicolumn{2}{c}{$|\in|<\mathbf{0 . 1}$ paths } & \multicolumn{2}{c}{$|\in|<\mathbf{0 . 0 1}$ paths } \\
\hline $\mathbf{k}$ & LN & IG & LN & IG \\
\hline 1 & $30.8 \%$ & $29.9 \%$ & $2.7 \%$ & $3.0 \%$ \\
10 & $80.1 \%$ & $78.4 \%$ & $12.2 \%$ & $9.5 \%$ \\
100 & $99.7 \%$ & $99.9 \%$ & $32.7 \%$ & $33.4 \%$ \\
1000 & $100 \%$ & $100 \%$ & $78.2 \%$ & $78.9 \%$ \\
10000 & $100 \%$ & $100 \%$ & $100 \%$ & $100 \%$ \\
\hline
\end{tabular}


FIGURE 3. HEDGING PORTFOLIO: OPTION NOT EXERCISED

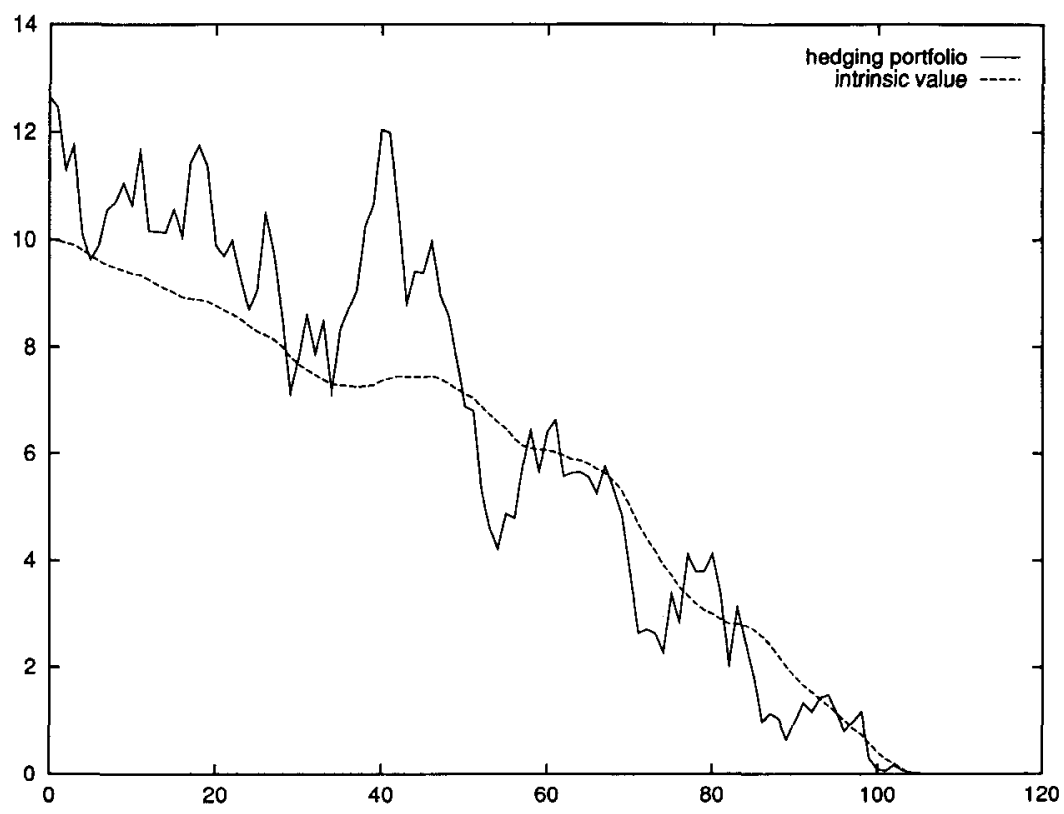

We are thus entitled to conclude that, as long as the hedging portfolio is updated sufficiently often, the approximation is of extremely good quality. The results presented in Table 3 provide a far stronger assessment of the quality of the approximation than mere Monte-Carlo simulation at a particular time. It is to be noted that this assessment is indeed strong since the trajectories have been generated with a value of the stock mean rate of return $a$ different from the risk-free interest rate $r$ (the independence of the price from $a$, guaranteed by the theory, could have been broken by the approximation).

\subsection{The limiting portfolio}

One interesting question is to know the value that the stock component of the hedging portfolio approaches when time approaches maturity. For a standard Black-Scholes option with payoff at maturity $\max \{S(T)-K, 0\}$, it is intuitive that the portfolio should ultimately be made of one share of stock if the option ends up in the money and nothing if the option ends up out of the money. Indeed, if the buyer of the option is to exercise the right to buy at a strike price lower than the current price of the stock, the seller of the option will have the share of stock at hand and will use the exercise price to cover the short position in the bond.

For Asian options, the intuition is not as clear since the comparison takes place with the arithmetic average of the last $n$ quoted prices. A priori the ultimate stock component of the portfolio might be path dependent. An analysis of many graphs of the type presented in the previous subsection shows that it is not the case. The intrinsic value of 
FIGURE 4. COMPONENTS OF PORTFOLIO: OPTION NOT EXERCISED

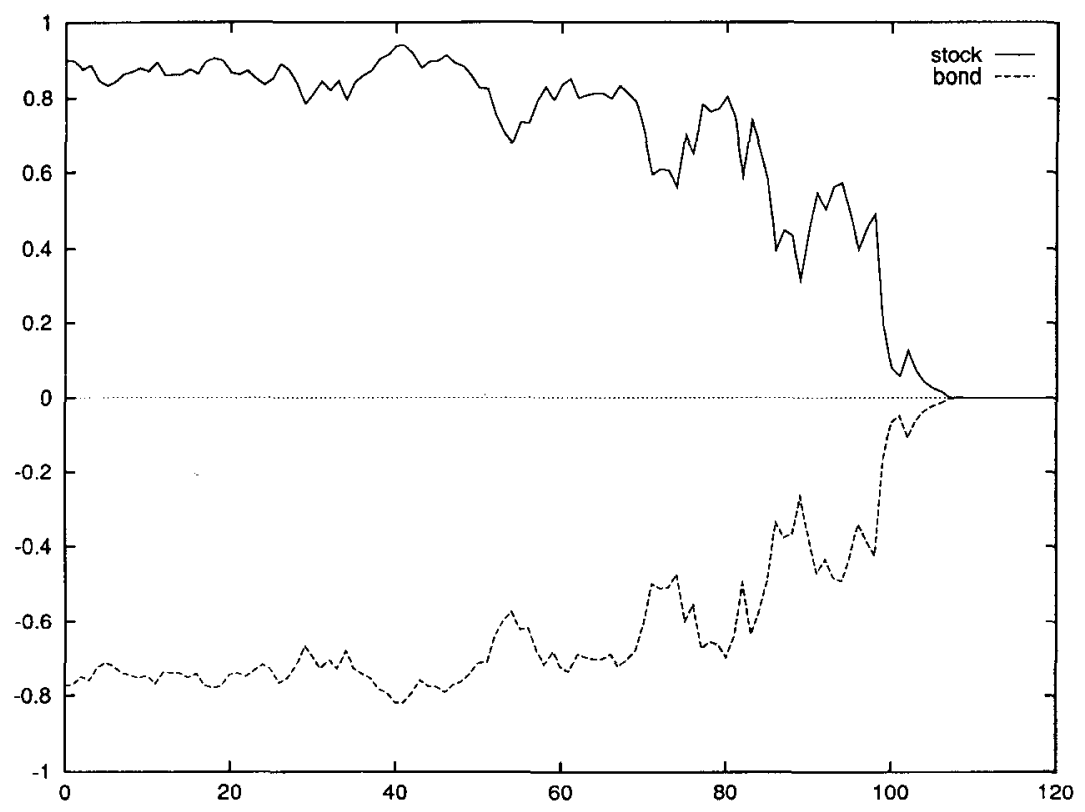

the option is $(S(T-n+1)+\ldots+S(T-1)+x) / n-K$ immediately before maturity if the option is to end up in the money and 0 otherwise. If we are allowed to interchange the differentiation process with the limit process, we get that $\xi=1 / n$ if the option ends up in the money and $\xi=0$ otherwise. We proceed now to take this limit directly on our expression for $\xi$.

What we need is the limit of (38) or (39) when $t$ approaches $T$. Thus $\bar{t}=T-1$ and, after some algebraic manipulations, we see from (13) and (14) that

$$
\begin{aligned}
& \lim _{t \rightarrow T} m_{1}(x, 1-t+\bar{t} ; r, b, T-\bar{t})=x, \\
& \lim _{t \rightarrow T} m_{2}(x, 1-t+\bar{t} ; r, b, T-\bar{t})=x^{2} .
\end{aligned}
$$

Hence, from (21) and (22),

$$
\begin{aligned}
& \lim _{t \rightarrow T} \mu(x, 1-t+\bar{t} ; r, b, T-\bar{t})=\ln x, \\
& \lim _{t \rightarrow T} \sigma(x, 1-t+\bar{t} ; r, b, T-\bar{t})^{2}=0 .
\end{aligned}
$$

In the limit when $t \rightarrow T$, the condition $\mu-\ln K^{\prime}>0$ is thus equivalent to $S(T)>n K-$ $(S(T-1)+\ldots+S(T-n+1))$, i.e. the option ends up in the money. Similarly, $\mu-\ln K^{\prime}$ $<0$ is equivalent to the option ending out of the money. Using $\Phi(+\infty)=1, \Phi(-\infty)=$ 0 , we obtain

$$
\lim _{t \rightarrow T} \xi(x, t ; r, b, n, T, K)=\left\{\begin{array}{r}
1 / n: \text { in the money, } \\
0: \text { out of the money. }
\end{array}\right.
$$


(Note that the limit in (39) is immediate.) These ultimate values of $\xi$ are indeed the values appearing in Figures 2 and 4.

\section{THE REPLICATING STRATEGY IN THE INVERSE GAUSSIAN APPROXIMATION}

Since the conceptual steps for the Inverse Gaussian approximation are identical to those for the Lognormal approximation, we just cite the results and indicate the computational tricks.

\subsection{The case $t<T-n+1$}

A straightforward integration (again well-known from the computation of Stop-Loss premiums) gives

$$
u(x, t ; r, b, n, T, K)=e^{-r(T-t)}\left[(\rho-K) \Phi\left(\frac{\rho-K}{\sqrt{\beta K}}\right)+(\rho+K) e^{2 \rho / \beta} \Phi\left(-\frac{\rho+K}{\sqrt{\beta K}}\right)\right],
$$

with the parameters $\rho$ and $\beta$ evaluated at $(x, T-t-n+1 ; r, b, n)$ (see (23), (24)). We easily see that

$$
\begin{aligned}
& \frac{\partial \rho}{\partial x}=\frac{\rho}{x}, \\
& \frac{\partial \beta}{\partial x}=\frac{\beta}{x},
\end{aligned}
$$

and, after some simplifications, we get

$$
\xi(x, t ; r, b, n, T, K)=e^{-r(T-t)} \frac{\rho}{x}\left[\Phi\left(\frac{\rho-K}{\sqrt{\beta K}}\right)+e^{2 \rho / \beta} \Phi\left(-\frac{\rho+K}{\sqrt{\beta K}}\right)\right],
$$

with the parameters $\rho$ and $\beta$ evaluated at $(x, T-t-n+1 ; r, b, n)$.

\subsection{The case $t \geq T-n+1, t$ non-integral}

The situation is the same as for the Lognormal approximation and we have

$$
\xi(x, t ; r, b, n, T, K)=e^{-r(T-t)} \frac{T-\bar{t}}{n} \frac{\rho}{x}\left[\Phi\left(\frac{\rho-K^{\prime}}{\sqrt{\beta K^{\prime}}}\right)+e^{2 \rho / \beta} \Phi\left(-\frac{\rho+K^{\prime}}{\sqrt{\beta K^{\prime}}}\right)\right],
$$

if $K^{\prime}>0$, and

$$
\xi(x, t ; r, b, n, T, K)=e^{-r(T-t)} \frac{T-\bar{t}}{n} \frac{\rho}{x},
$$

if $K^{\prime} \leq 0$. In both equations the parameters $\rho$ and $\beta$ are evaluated at $(x, 1-t+\bar{t} ; r, b, T-\bar{t})$. 


\subsection{The case $t \geq T-n+1, t$ integral}

Again proceeding as in the Lognormal case, we get

$$
\begin{gathered}
\xi(x, t ; r, b, n, T, K)=e^{-r(T-t)} \frac{T-t}{n} \\
{\left[\left(\frac{\rho}{x}+\frac{1}{T-t}\right) \Phi\left(\frac{\rho-K^{\prime}}{\sqrt{\beta K^{\prime}}}\right)+\left(\frac{\rho}{x}-\frac{1}{T-t}\right) e^{2 \rho / \beta} \Phi\left(-\frac{\rho+K^{\prime}}{\sqrt{\beta K^{\prime}}}\right)\right],}
\end{gathered}
$$

if $K^{\prime}>0$, and

$$
\xi(x, t ; r, b, n, T, K)=e^{-r(T-t)} \frac{T-t}{n}\left(\frac{\rho}{x}+\frac{1}{T-t}\right),
$$

if $K^{\prime} \leq 0$. In both equations the parameters $\rho$ and $\beta$ are evaluated at $(x, 1 ; r, b, T-t)$.

\subsection{Examples}

The same trajectories have been considered as for the Lognormal approximation. The formulae for the hedging portfolio using the Inverse Gaussian approximation present a numerical difficulty due to the factor $e^{2 \rho / \beta}$. For the numerical values used in section 4.4, the exponential factor blows up on most computer systems. One way to get rid of this problem is to use the Mill ratio for the tail of the Normal cumulative probability function (see for instance Resnick (1992), p. 487):

$$
\begin{aligned}
1-\Phi(x) & \sim \frac{\varphi(x)}{x} \quad \text { as } \mathrm{x} \rightarrow+\infty, \\
\Phi(x) & \sim-\frac{\varphi(x)}{x} \quad \text { as } \mathrm{x} \rightarrow-\infty,
\end{aligned}
$$

where $\varphi$ is the probability density of a standardized Normal variable. This means that

$$
e^{2 \rho / \beta} \Phi\left(-\frac{\rho+K}{\sqrt{\beta K}}\right) \sim \frac{\sqrt{\beta K}}{\rho+K} \varphi\left(\frac{\rho-K}{\sqrt{\beta K}}\right) \text { as } \beta \rightarrow 0 \text {. }
$$

This approximation is used as soon as the ratio $2 \rho / \beta$ attains a value that makes the exponential function blow up on the computer system under use.

With this additional feature, the resulting figures are completely undistinguishable from those presented in section 4.4: we could have given them on the same figures, but the reader would not have noticed them.

As far as the numerical analysis of the error of the hedging portfolio is concerned, the corresponding values are also given in the IG columns of Table 3. It is clear from those values that the Inverse Gaussian approximation should be considered as efficient as the Lognormal approximation. All the comparisons used in this paper do not allow for a preference between the two approximations. 


\subsection{The limiting portfolio}

Using (52) together with

$$
\begin{aligned}
& \lim _{t \rightarrow T} \rho(x, 1-t+\bar{t} ; r, b, T-\bar{t})=x, \\
& \lim _{t \rightarrow T} \beta(x, 1-t+\bar{t} ; r, b, T-\bar{t})=0,
\end{aligned}
$$

we arrive at the same conclusion as in the Lognormal approximation:

$$
\lim _{t \rightarrow T} \xi(x, t ; r, b, n, T, K)=\left\{\begin{array}{c}
1 / n: \text { in the money, } \\
0: \text { out of the money. }
\end{array}\right.
$$

\section{CONCLUSION}

This paper gives explicit formulae for building the hedging portfolio for Asian (arithmetic average) options. The formulae are based on the approximation of the arithmetic average of correlated Lognormal variables by either a Lognormal or an Inverse Gaussian variable. Replicating strategies (a key ingredient of option pricing theory) have been built with the help of these formulae and have been used as a measure of the quality of the approximations. The numerical examples given in section 4.4 and 5.4 provide a strong assessment of the good quality of both approximations. Since the distribution of the average is not known, it is important to consider all relevant approximations. The comparisons between the two approximations show that they are equally efficient.

\section{ACKNOWLEDGEMENT}

The author wishes to thank Jacques Rioux for useful discussions and the anonymous referees for constructive remarks that helped to produce a better paper.

\section{REFERENCES}

Bergman, YaAKov Z. (1985) "Pricing Path Contingent Claims". Research in Finance 5, 229-241.

Bowers, N. L., Gerber, H. U., Hickman, J. C., Jones, D. A., And Nesbitt, C. J. (1986) Actuarial Mathematics. Society of Actuaries.

Geman, Hélyette and Yor, Marc (1993) "Bessel Processes, Asian Options, and Perpetuities". Mathematical Finance 3, 349-375.

HARRISON, J. M. AND KREPS, D. M. (1979) "Martingales and arbitrage in multiperiod securities markets". Journal of Economic Theory 20, 381-408.

HARRISON, J. M. AND Pliska, S. (1981) "Martingales and stochastic integrals in the theory of continuous trading". Stochastic Processes and Their Applications 11, 215-260.

Hogg, R. V. AND KLugman, S.A. (1984) Loss Distributions. Wiley.

Johnson, Norman L. AND kotz, SAMUel (1970) Distributions in Statistics: Continuous Univariate Distributions-1. Wiley. 
Kemna, A. G. Z. AND Vorst, A. C. F. (1990) "A pricing method for options based on average asset value". Journal of Banking and Finance 14, 113-129.

LEVY, EDMOND (1992) "Pricing European average rate currency options". Journal of International Money and Finance 11, 474-491.

RESNICK, STANLEy (1992) Adventures in Stochastic Processes. Birkhäuser.

Turnbull, S. M. and Wakeman, L. M. (1991) "A Quick Algorithm for Pricing European Average Options". Journal of Financial and Quantitative Analysis 26, 377-389.

VORST, TON (1992) "Prices and Hedge Ratios of Average Exchange Rate Options". International Review of Financial Analysis 1, 179-193.

Michel JACQUES

École d'actuariat, Université Laval

Québec, Canada G1K 7P4

mjacques@act.ulaval.ca 